\title{
Short-Term Effects of Acupuncture on Open-Angle Glaucoma in Retrobulbar Circulation: Additional Therapy to Standard Medication
}

\author{
Shin Takayama, ${ }^{1}$ Takashi Seki, ${ }^{1}$ Toru Nakazawa, ${ }^{2}$ Naoko Aizawa, ${ }^{2}$ \\ Seri Takahashi, ${ }^{2}$ Masashi Watanabe, ${ }^{1}$ Masayuki Izumi, ${ }^{1}$ Soichiro Kaneko, ${ }^{1}$ \\ Tetsuharu Kamiya, ${ }^{1}$ Ayane Matsuda, ${ }^{1}$ Akiko Kikuchi, ${ }^{1}$ Tomoyuki Yambe, ${ }^{3}$ \\ Makoto Yoshizawa, ${ }^{4}$ Shin-ichi Nitta, ${ }^{3}$ and Nobuo Yaegashi ${ }^{1}$ \\ ${ }^{1}$ Department of Traditional Asian Medicine, Graduate School of Medicine, Tohoku University, \\ 1-1 Seiryo-machi, Aoba-ku, Sendai, Miyagi 980-8574, Japan \\ ${ }^{2}$ Department of Ophthalmology and Visual Science, Graduate School of Medicine, Tohoku University, Sendai 980-8574, Japan \\ ${ }^{3}$ Institute of Development, Aging and Cancer, Tohoku University, Sendai 980-8575, Japan \\ ${ }^{4}$ Research Division on Advanced Information Technology, Cyberscience Center, Tohoku University, Japan
}

Correspondence should be addressed to Takashi Seki, t-seki@m.tohoku.ac.jp

Received 29 October 2010; Revised 7 December 2010; Accepted 11 January 2011

Copyright () 2011 Shin Takayama et al. This is an open access article distributed under the Creative Commons Attribution License, which permits unrestricted use, distribution, and reproduction in any medium, provided the original work is properly cited.

\begin{abstract}
Background. The relation between glaucoma and retrobulbar circulation in the prognosis has been indicated. Purpose. To investigate the effects of acupuncture on retrobulbar circulation in open-angle glaucoma (OAG) patients. Methods. Eleven OAG patients (20 eyes with OAG) who were treated by topical antiglaucoma medications for at least 3 months were enrolled. Acupuncture was performed once at acupoints BL2, M-HN9, ST2, ST36, SP6, KI3, LR3, GB20, BL18, and BL23 bilaterally. Retrobulbar circulation was measured with color Doppler imaging, and intraocular pressure (IOP) was also measured at rest and one hour after rest or before and after acupuncture. Results. The $\Delta$ value of the resistive index in the short posterior ciliary artery $(P<.01)$ and the $\Delta$ value of IOP $(P<.01)$ were decreased significantly by acupuncture compared with no acupuncture treatment. Conclusions. Acupuncture can improve the retrobulbar circulation and IOP, which may indicate the efficacy of acupuncture for OAG.
\end{abstract}

\section{Introduction}

Glaucoma is one of the causes of blindness [1] and the Tajimi Study showed that the prevalence of primary open-angle glaucoma (OAG) was 3.9\% in Japan [2]. The main treatment strategy of glaucoma is to control the intraocular pressure (IOP) [3]. Although IOP reduction is currently the main target for the treatment of glaucoma, treatment modalities that enhance retrobulbar hemodynamics in addition to reducing IOP may have a beneficial effect on the glaucoma therapy. It has been reported that glaucoma is associated with reduction in the blood flow velocity and elevation of the resistive index (RI) in the retrobulbar vessels [47]. It has also been reported that patients with OAG have impaired hemodynamics in ophthalmic circulation [8-10].
The impaired ocular circulation contributes to the progression of glaucomatous damage [11-13]. Therefore, new drugs or interventions that improve ocular hemodynamics may be preferable.

Recently, acupuncture has been widely applied to treat several conditions such as neck pain, shoulder pain, lumbar pain, headache, and hypertension in Asian and Western countries, and it has also been found to be effective for many conditions in several randomized trials [1420]. Acupuncture has also been used for the treatment of ocular diseases, including glaucoma, in traditional Chinese medicine [21]. We have shown that acupuncture therapy added to the standard medication could affect the IOP level in eyes with normal-tension glaucoma [22], and several other studies have demonstrated that 
acupuncture improves choroidal blood flow in the eye [2325].

We have already reported that color Doppler imaging (CDI) by ultrasound is suitable for measuring the blood flow change in several organs during traditional Chinese medicine therapy [26-30]. The real-time and noninvasive hemodynamic measurement with CDI has been applied for measuring the retrobulbar vessel hemodynamics, and the reproducibility has already been shown [31]. In this study, we evaluate the hemodynamic changes in retrobulbar vessels by CDI to investigate the effect of acupuncture on OAG eyes.

\section{Subjects}

After the ethics committee approved the study, 11 patients diagnosed with OAG (20 eyes with OAG) were enrolled in this study. The patients received standard medical treatment for at least 3 months. The patients who had an experience of laser trabeculoplasty, any ocular surgery, or inflammation within the past year were excluded in the present study.

\section{Methods}

3.1. Acupuncture. On the trial days, the patients arrived under regular medications. They received acupuncture therapy as follows in the morning. The acupoints were selected on the basis of the principles of traditional Chinese medicine. Acupuncture was performed for $15 \mathrm{~min}$ using disposable stainless steel needles $(0.16 \mathrm{~mm}$ or $0.20 \mathrm{~mm} \times 40 \mathrm{~mm}$; Seirin Co. Ltd., Shizuoka, Japan) at acupoints Cuanzhu (BL2), Taiyang (M-HN9), Sibai (ST2), Zusanli (ST36), Sanyinjiao (SP6), Taixi (KI3), and Taichong (LR3) bilaterally while the patient was in the supine position and at acupoints Fengchi (GB20), Ganshu (BL18), and Shenshu (BL23) bilaterally while the patient was in the prone position for $15 \mathrm{~min}$. Each needle was simply inserted without any intention of eliciting specific responses (e.g., de-qi feelings) to a depth of approximately $20 \mathrm{~mm}$ at acupoints ST36, SP6, KI3, GB20, BL18, and BL23. For acupoints BL2, M-HN9, ST2, and LR3, the needles were inserted to a depth of approximately 3$10 \mathrm{~mm}$. Neither needle manipulation techniques nor other auxiliary interventions were used. Five licensed acupuncturists and one physician-acupuncturist with over 5 years of acupuncture experience administered the acupuncture treatment.

3.2. Measurements. To minimize the effects of diurnal variation, all measurements were recorded at the same time of the day (between $10 \mathrm{AM}$ and $11 \mathrm{AM}$ ) for each patient by the same examiner. As a control, the subjects received the measurements of the systemic hemodynamics, retrobulbar vessel hemodynamics, and IOP that were performed at rest and one hour after rest. One month later, they received the same measurements before and after acupuncture treatment. The systemic hemodynamics was measured by an oscillometer and the hemodynamics in retrobulbar vessels was measured by ultrasound (LOGIQ e, GE Healthcare, Tokyo, Japan). The ultrasound measurements were performed after 10-minute
TABLE 1: Characteristic data of the patients with open-angle glaucoma.

\begin{tabular}{lc}
\hline Variable & Value \\
\hline Number of patients & 11 \\
Age (years) & $63 \pm 11$ \\
Sexuality (male, female) & $(1,10)$ \\
Number of eyes with glaucoma & 20 \\
Best corrected visual acuity & $1.1 \pm 0.3$ \\
Spherical equivalent (D) & $-1.6 \pm 3.2$ \\
Humphrey automated perimeter & \\
$\quad$ Mean deviation (dB) & $11.5 \pm 7.8$ \\
$\quad$ Pattern standard deviation (dB) & $10.2 \pm 4.5$ \\
$\quad$ OCT RNFL thickness $(\mu \mathrm{m})$ & $70.5 \pm 21.8$ \\
The number of topical medications & 1 \\
$\quad$ None & 4 \\
One kind & 1 \\
Two kinds & 5 \\
$\quad$ More than three kinds & \\
\hline
\end{tabular}

rest in an air-conditioned room, avoiding any pressure on the eye, with the patients in the supine position. CDI was performed with a $13 \mathrm{MHz}$ linear transducer for retrobulbar vessels such as the ophthalmic artery (OA), central retinal artery (CRA), and short posterior ciliary artery (SPCA). The OA was examined approximately $20 \mathrm{~mm}$ behind the globe (Figure 1(a)), the CRA was examined within $5 \mathrm{~mm}$ of the retrolaminar portion of the optic nerve (Figure 1(b)), and the temporal SPCA was examined approximately 5$10 \mathrm{~mm}$ behind the globe (Figure 1(c)). All blood flow velocity waveforms were measured at the corrected Doppler angle. Resistive index (RI: (peak systolic velocity - end-diastolic velocity)/peak systolic velocity) was also measured in each retrobulbar vessel.

3.3. Statistical Analysis. Statistical analysis was performed with the SPSS software (version 16.0, SPSS Japan Inc., Tokyo, Japan). The parameters between before and after acupuncture or between control and acupuncture were compared by paired $t$-test.

\section{Results}

Table 1 shows the characteristics of the subjects. One male and ten female glaucoma patients with a mean age of $63 \pm 11$ years were observed. The systemic hemodynamic parameters including heart rate, blood pressure, and IOP are shown in Table 2. The blood pressure and heart rate did not change significantly by acupuncture.

The IOP level significantly decreased by acupuncture compared with before acupuncture $(P<.05)$. The $\Delta$ value of IOP also significantly decreased by acupuncture compared with control $(P<.01)$ (Table 2$)$.

Retrobulbar vessel RI in the OA, CRA, and SPCA is shown in Table 3. The RI in the CRA and SPCA decreased 


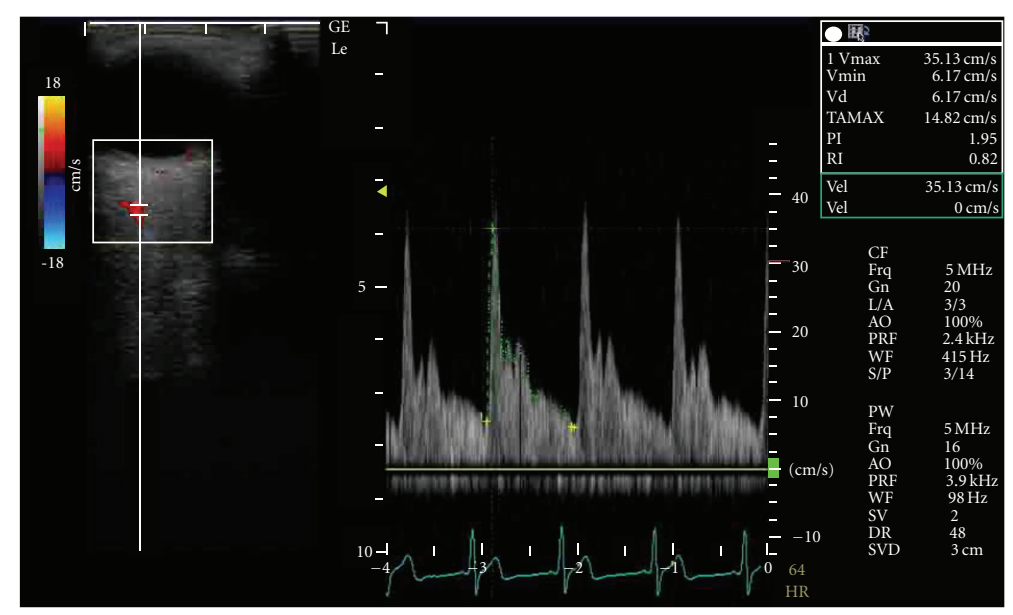

(a)

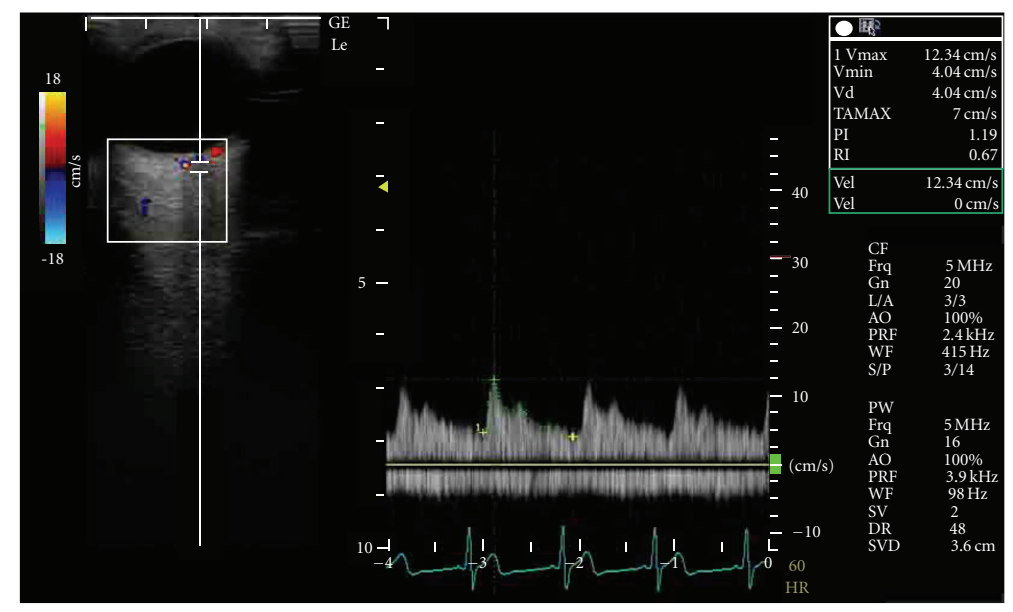

(b)

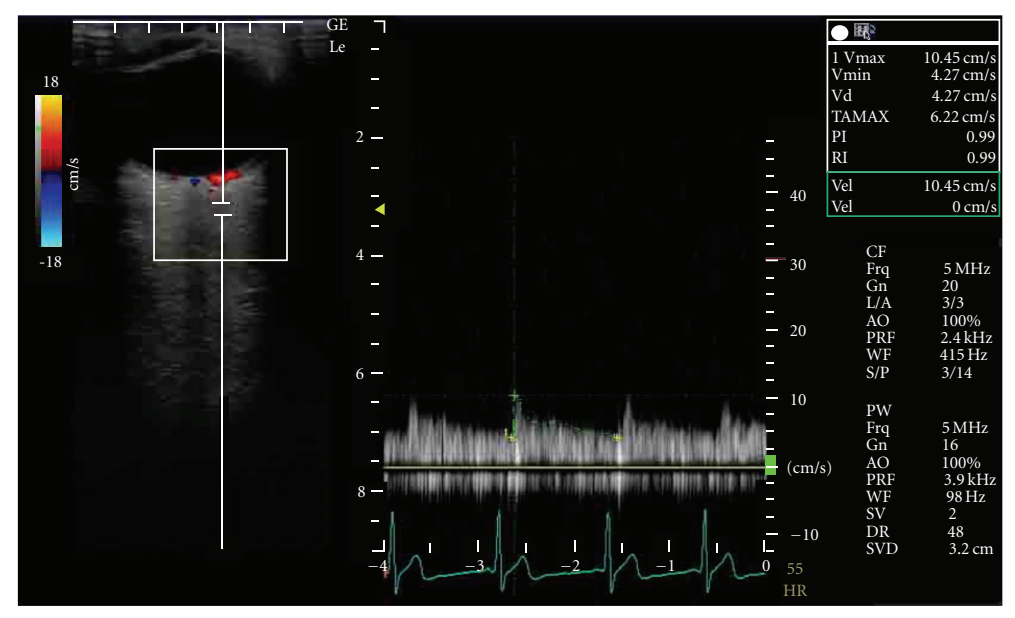

(c)

FIGURE 1: Horizontal scans by color Doppler imaging through the globe showing the (a) ophthalmic artery, (b) central retinal artery, and (c) short posterior ciliary artery. 
TABLE 2: Blood pressure, heart rate, and intraocular pressure in control and acupuncture therapy. The values represent the mean and SD. ${ }^{*} P<.05,{ }^{* *} P<.01$ versus rest or before acupuncture. ${ }^{\dagger} P<.05,{ }^{\dagger \dagger} P<.01$ versus control.

\begin{tabular}{|c|c|c|c|c|c|c|}
\hline \multirow{2}{*}{ Parameter } & \multicolumn{3}{|c|}{ Control } & \multicolumn{3}{|c|}{ Acupuncture } \\
\hline & Rest & After 1 hour & $\Delta$ value & Before & After & $\Delta$ value \\
\hline Systole blood pressure ( $\mathrm{mm} \mathrm{Hg}$ ) & $116.4 \pm 10.0$ & $119.8 \pm 7.6$ & $3.4 \pm 7.4$ & $124.5 \pm 12.9$ & $122.6 \pm 9.7$ & $-1.1 \pm 7.9$ \\
\hline Diastolic blood pressure (mm Hg) & $69.8 \pm 6.5$ & $68.6 \pm 3.9$ & $-1.0 \pm 9.4$ & $74.5 \pm 5.4$ & $72.0 \pm 2.9$ & $-3.0 \pm 5.5$ \\
\hline Heart rate (beats/min) & $61.5 \pm 7.3$ & $60.1 \pm 8.1$ & $-2.5 \pm 3.8$ & $61.7 \pm 8.5$ & $60.3 \pm 10.4$ & $-2.4 \pm 5.5$ \\
\hline Intraocular pressure (mm Hg) & $16.0 \pm 4.1$ & $17.1 \pm 4.2^{* *}$ & $1 \pm 0.9$ & $17.0 \pm 5.0$ & $16.0 \pm 4.3^{*}$ & $-1 \pm 1.9^{\dagger \dagger}$ \\
\hline
\end{tabular}

TABLE 3: Resistive index (RI) in the ophthalmic artery, central retinal artery, and short posterior ciliary artery. The values represent the mean and SD. ${ }^{*} P<.05,{ }^{* *} P<.01$ versus before acupuncture. ${ }^{\dagger} P<.05,{ }^{\dagger \dagger} P<.01$ versus control.

\begin{tabular}{lcccccc}
\hline Resistive index & \multicolumn{3}{c}{ Control } & \multicolumn{3}{c}{ Acupuncture } \\
& Rest & After 1 hour & $\Delta$ value & Before & After & $\Delta$ value \\
\hline Ophthalmic artery & $0.74 \pm 0.04$ & $0.75 \pm 0.05$ & $0.006 \pm 0.037$ & $0.74 \pm 0.04$ & $0.74 \pm 0.04$ & $-0.006 \pm 0.036$ \\
Central retinal artery & $0.75 \pm 0.09$ & $0.72 \pm 0.03$ & $-0.027 \pm 0.085$ & $0.72 \pm 0.05$ & $0.68 \pm 0.04^{*}$ & $-0.036 \pm 0.059$ \\
Short posterior ciliary artery & $0.68 \pm 0.05$ & $0.68 \pm 0.04$ & $0.004 \pm 0.038$ & $0.67 \pm 0.04$ & $0.64 \pm 0.06^{*}$ & $-0.032 \pm 0.054^{\dagger \dagger}$ \\
\hline
\end{tabular}

significantly by acupuncture compared with before acupuncture $(P<.05)$. The $\Delta$ value of RI in the SPCA also significantly decreased by acupuncture compared with control $(P<.01)$ (Table 3).

\section{Discussion}

To our best knowledge, this is the first report on hemodynamic change in retrobulbar vessels related to acupuncture in OAG eyes. The present findings suggest that acupuncture can alter vessel resistance in the SPCA, even though the eyes are treated with standard medications.

The OA originates from the internal carotid artery. The CRA and SPCA are the ocular branches of the OA [32]. The CRA supplies blood to the retina and SPCA, to the choroid. CDI by ultrasound is useful for the measurement of the blood flow in various vessels in real time. Since it is impossible to determine the diameter of very small retrobulbar vessels, CDI cannot directly measure blood flow volume. However, the decrease of the distal vascular resistance in the SPCA indicates an increase of the blood flow in the choroid. We have already reported that acupuncture could increase the blood flow volume in the upper limb without an increase in the cardiac output, and the increased reaction in the blood flow was mediated by the decrease in the vascular resistance on the basis of the decreased vascular tone [30]. The mechanisms by which acupuncture can alter retrobulbar vessel circulation are still unclear. However, it has been reported that the blood flow in the eye is controlled by sympathetic and parasympathetic nerves, and it is related with the release of nitric oxide or calcitonin gene-related peptide $[33,34]$; it has also been reported that the regulation of regional blood flow by somatic afferent stimulation is based on somatoautonomic reflex mechanisms in the choroidal blood flow of the eyeball [34]. The hemodynamic changes in the SPCA by acupuncture may be related with these mechanisms. Reduced blood flow velocities and increased vascular resistance in the retrobulbar arteries appear to be a risk factor for glaucoma progression [35-38]. Thus, acupuncture may be applied for additional therapy to treat OAG.

We should view these results cautiously because the present study was a case series study and intervention was provided only once. Longer observation of acupuncture therapy is needed to investigate the progression of glaucomatous damage associated with impaired ocular circulation.

\section{Conclusions}

The vessel resistance in the SPCA and the IOP level were decreased by acupuncture in OAG eyes. Acupuncture can affect the retrobulbar circulation and IOP despite the administration of standard medication. The present study implies the possibility that acupuncture is effective for OAG with standard medication.

\section{Acknowledgment}

This work was supported by Health and Labour Sciences Research Grants for Clinical Research from the Japanese Ministry of Health, Labour and Welfare.

\section{References}

[1] K. Nakae, K. Masuda, T. Aneo et al., "Wagakuni ni okeru shiryokushougai no genjou," Research Committee on Chorioretinal Degenerations and Optic Atrophy, the Ministry of Health, Labour and Welfare of Japan, vol. 17, pp. 263-276, 2005.

[2] A. Iwase, Y. Suzuki, M. Araie et al., "The prevalence of primary open-angle glaucoma in Japanese: the Tajimi study," Ophthalmology, vol. 111, no. 9, pp. 1641-1648, 2004.

[3] R. N. Weinreb and P. Tee Khaw, "Primary open-angle glaucoma," The Lancet, vol. 363, no. 9422, pp. 1711-1720, 2004.

[4] C. Akarsu and M. Y. K. Bilgili, "Color Doppler imaging in ocular hypertension and open-angle glaucoma," Graefe's 
Archive for Clinical and Experimental Ophthalmology, vol. 242, no. 2, pp. 125-129, 2004.

[5] V. P. Costa, A. Harris, E. Stefánsson et al., "The effects of antiglaucoma and systemic medications on ocular blood flow," Progress in Retinal and Eye Research, vol. 22, no. 6, pp. 769-805, 2003.

[6] H. J. Kaiser, A. Schoetzau, D. Stumpfig, and J. Flammer, "Blood-flow velocities of the extraocular vessels in patients with high- tension and normal-tension primary open-angle glaucoma," American Journal of Ophthalmology, vol. 123, no. 3, pp. 320-327, 1997.

[7] S. J. A. Rankin, "Color Doppler imaging of the retrobulbar circulation in glaucoma," Survey of Ophthalmology, vol. 43, no. 1, pp. S176-S182, 1999.

[8] I. Stalmans, A. Harris, S. Fieuws et al., "Color Doppler imaging and ocular pulse amplitude in glaucomatous and healthy eyes," European Journal of Ophthalmology, vol. 19, no. 4, pp. 580-587, 2009.

[9] I. Janulevičiene, I. Sliesoraityte, B. Siesky, and A. Harris, "Diagnostic compatibility of structural and haemodynamic parameters in open-angle glaucoma patients," Acta Ophthalmologica, vol. 86, no. 5, pp. 552-557, 2008.

[10] N. Plange, M. Kaup, O. Arend, and A. Remky, "Asymmetric visual field loss and retrobulbar haemodynamics in primary open-angle glaucoma," Graefe's Archive for Clinical and Experimental Ophthalmology, vol. 244, no. 8, pp. 978-983, 2006.

[11] M. Satilmis, S. Orgül, B. Doubler, and J. Flammer, "Rate of progression of glaucoma correlates with retrobulbar circulation and intraocular pressure," American Journal of Ophthalmology, vol. 135, no. 5, pp. 664-669, 2003.

[12] J. Schumann, S. Orgül, K. Gugleta, B. Dubler, and J. Flammer, "Interocular difference in progression of glaucoma correlates with interocular differences in retrobulbar circulation," American Journal of Ophthalmology, vol. 129, no. 6, pp. 728-733, 2000.

[13] Y. Yamazaki and S. M. Drance, "The relationship between progression of visual field defects and retrobulbar circulation in patients with glaucoma," American Journal of Ophthalmology, vol. 124, no. 3, pp. 287-295, 1997.

[14] X. Xu, "Acupuncture in an outpatient clinic in China: a comparison with the use of acupuncture in North America," Southern Medical Journal, vol. 94, no. 1-10, pp. 813-816, 2001.

[15] V. Napadow and T. J. Kaptchuk, "Patient characteristics for outpatient acupuncture in Beijing, China," Journal of Alternative and Complementary Medicine, vol. 10, no. 3, pp. 565-572, 2004.

[16] C. M. Witt, S. Jena, B. Brinkhaus, B. Liecker, K. Wegscheider, and S. N. Willich, "Acupuncture for patients with chronic neck pain," Pain, vol. 125, no. 1-2, pp. 98-106, 2006.

[17] B. Brinkhaus, C. M. Witt, S. Jena et al., "Acupuncture in patients with chronic low back pain: a randomized controlled trial," Archives of Internal Medicine, vol. 166, no. 4, pp. 450457, 2006.

[18] D. Melchart, A. Streng, A. Hoppe et al., "Acupuncture in patients with tension-type headache: randomised controlled trial," British Medical Journal, vol. 331, no. 7513, pp. 376-379, 2005.

[19] C. Witt, B. Brinkhaus, S. Jena et al., "Acupuncture in patients with osteoarthritis of the knee: a randomised trial," The Lancet, vol. 366, no. 9480, pp. 136-143, 2005.

[20] K. Linde, A. Streng, S. Jürgens et al., "Acupuncture for patients with migraine: a randomized controlled trial," Journal of the American Medical Association, vol. 293, no. 17, pp. 2118-2125, 2005.
[21] Diseases of Eyes, Ears, Nose and Throat, vol. 681, Eastland Press, Seattle, Wash, USA, 1981.

[22] M. Kurusu, K. Watanabe, T. Nakazawa et al., "Acupuncture For Patients With Glaucoma,” Explore, vol. 1, no. 5, pp. 372-376, 2005.

[23] S. Naruse, K. Mori, M. Kurihara et al., "Chorioretinal blood flow changes following acupuncture between thumb and forefinger," Journal of Japanese Ophthalmological Society, vol. 104, no. 10, pp. 717-723, 2000.

[24] M. Shimura, S. Uchida, A. Suzuki, K. Nakajima, and Y. Aikawa, "Reflex choroidal blood flow responses of the eyeball following somatic sensory stimulation in rats," Autonomic Neuroscience, vol. 97, no. 1, pp. 35-41, 2002.

[25] J. J. Steinle, D. Krizsan-Agbas, and P. G. Smith, "Regional regulation of choroidal blood flow by autonomic innervation in the rat," American Journal of Physiology, vol. 279, no. 1, pp. R202-R209, 2000.

[26] S. Takayama, T. Seki, N. Sugita et al., "Radial artery hemodynamic changes related to acupuncture," Explore, vol. 6, no. 2, pp. 100-105, 2010.

[27] S. Takayama, T. Seki, M. Watanabe et al., "Changes of blood flow volume in the superior mesenteric artery and brachial artery with abdominal thermal stimulation," Evidence-Based Complementary and Alternative Medicine, vol. 17, pp. 1-9, 2009.

[28] S. Takayama, T. Seki, M. Watanabe et al., "The herbal medicine Daikenchuto increases blood flow in the superior mesenteric artery," Tohoku Journal of Experimental Medicine, vol. 219, no. 4, pp. 319-330, 2009.

[29] S. Takayama, T. Seki, M. Watanabe et al., "The effect of warming of the abdomen and of herbal medicine on superior mesenteric artery blood flow-a pilot study," Forschende Komplementarmedizin, vol. 17, no. 4, pp. 195-201, 2010.

[30] S. Takayama, T. Seki, M. Watanabe et al., "Brief effect of acupuncture on the peripheral arterial system of the upper limb and systemic hemodynamics in humans," Journal of Alternative and Complementary Medicine, vol. 16, no. 7, pp. 707-713, 2010.

[31] E. T. Matthiessen, O. Zeitz, G. Richard, and M. Klemm, "Reproducibility of blood flow velocity measurements using colour decoded Doppler imaging," Eye, vol. 18, no. 4, pp. 400405, 2004.

[32] S. S. Hayreh and R. Dass, "The ophthalmic artery II: intra orbital course," The British Journal of Ophthalmology, vol. 46, no. 3, pp. 165-185, 1962.

[33] A. K. Wiencke, H. Nilsson, P. J. Nielsen, and N. C. B. Nyborg, "Nonadrenergic noncholinergic vasodilation in bovine ciliary artery involves CGRP and neurogenic nitric oxide," Investigative Ophthalmology and Visual Science, vol. 35, no. 8, pp. 32683277, 1994.

[34] M. Shimura, S. Uchida, A. Suzuki, K. Nakajima, and Y. Aikawa, "Reflex choroidal blood flow responses of the eyeball following somatic sensory stimulation in rats," Autonomic Neuroscience, vol. 97, no. 1, pp. 35-41, 2002.

[35] D. Gherghel, S. Orgül, K. Gugleta, M. Gekkieva, and J. Flammer, "Relationship between ocular perfusion pressure and retrobulbar blood flow in patients with glaucoma with progressive damage," American Journal of Ophthalmology, vol. 130, no. 5, pp. 597-605, 2000.

[36] O. Zeitz, P. Galambos, L. Wagenfeld et al., "Glaucoma progression is associated with decreased blood flow velocities in the short posterior ciliary artery," British Journal of Ophthalmology, vol. 90, no. 10, pp. 1245-1248, 2006. 
[37] F. Galassi, A. Sodi, F. Ucci, G. Renieri, B. Pieri, and M. Baccini, "Ocular hemodynamics and glaucoma prognosis: a color Doppler imaging study," Archives of Ophthalmology, vol. 121, no. 12, pp. 1711-1715, 2003.

[38] A. Martínez and M. Sánchez, "Predictive value of colour Doppler imaging in a prospective study of visual field progression in primary open-angle glaucoma," Acta Ophthalmologica Scandinavica, vol. 83, no. 6, pp. 716-722, 2005. 


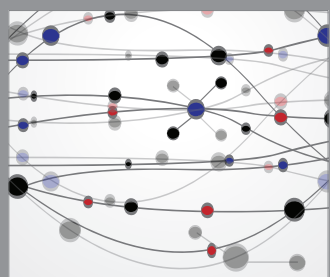

The Scientific World Journal
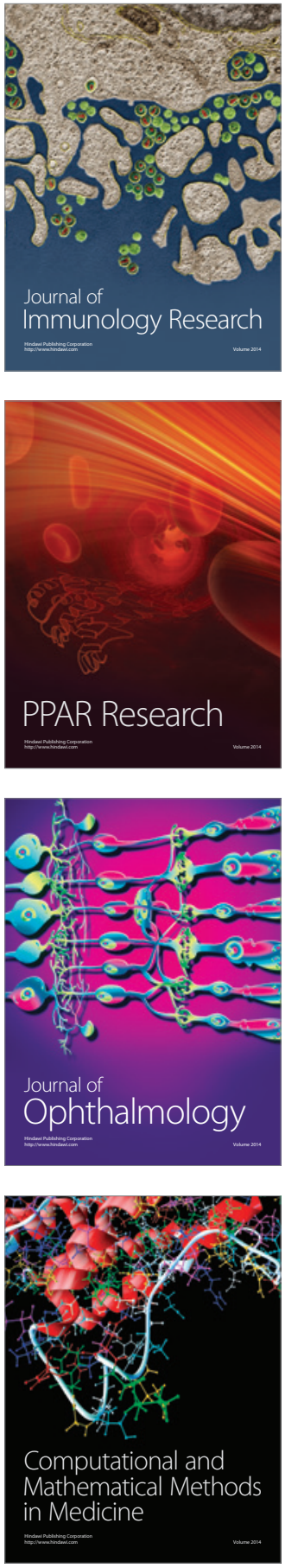

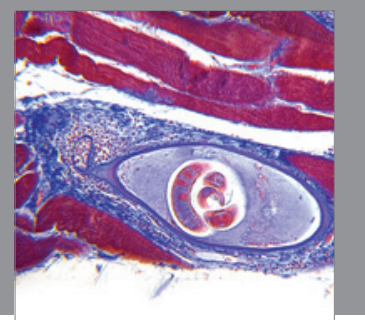

Gastroenterology

Research and Practice
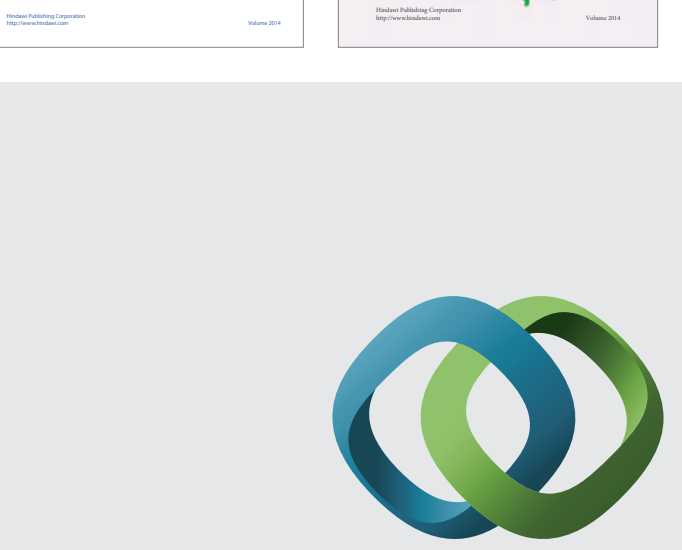

\section{Hindawi}

Submit your manuscripts at

http://www.hindawi.com
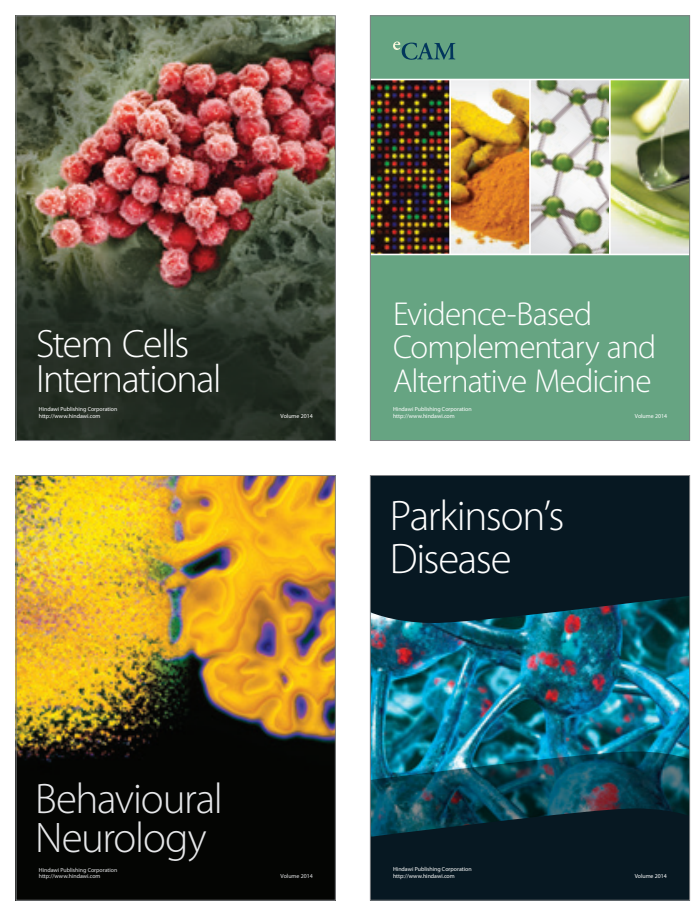

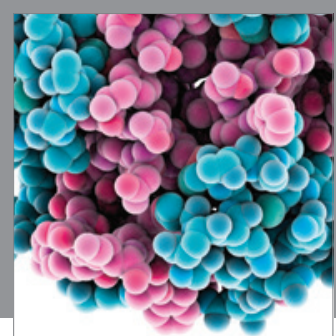

Journal of
Diabetes Research

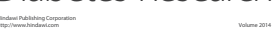

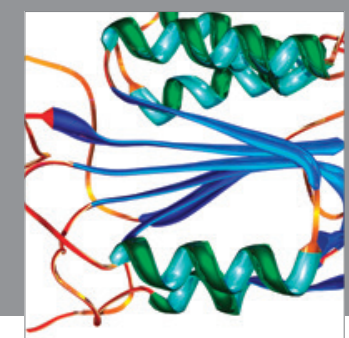

Disease Markers
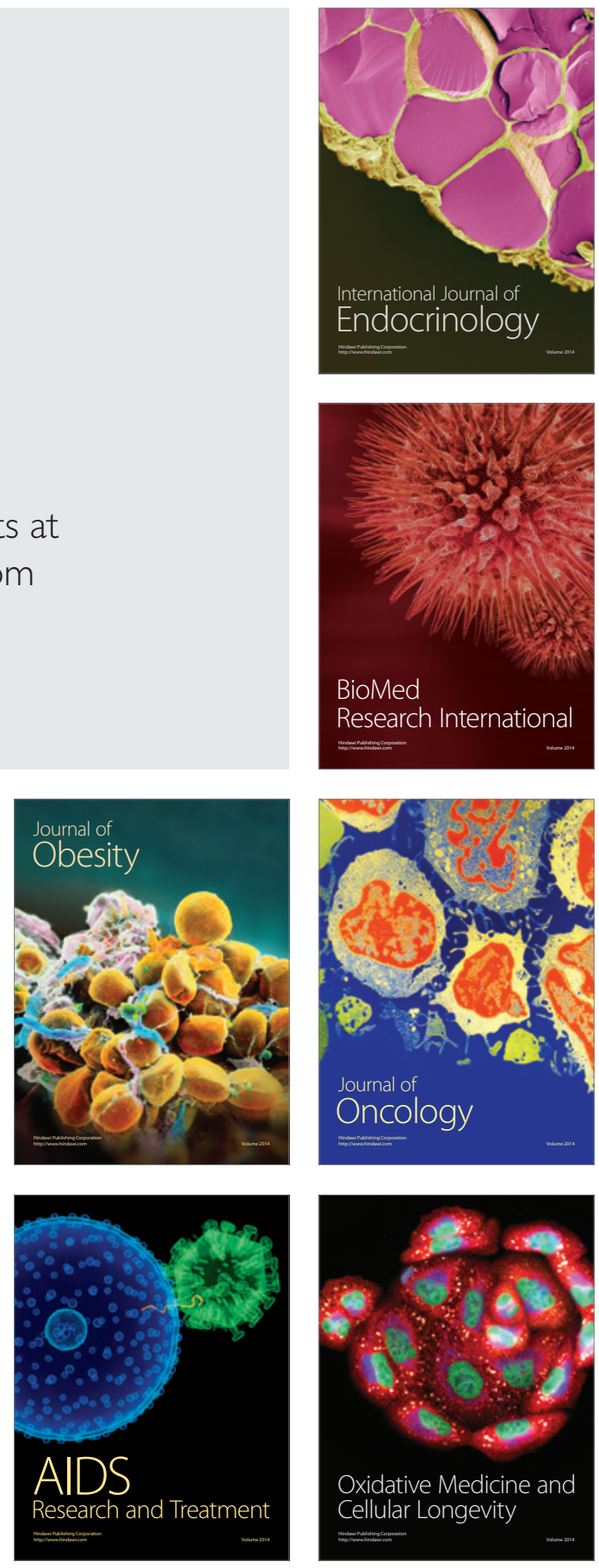